\title{
Anxiety and orienting tasks in picture recognition
}

\author{
JOHN H. MUELLER, DAVID J. MILLER, and JEFFREY L. HUTCHINGS \\ University of Missouri, Columbia, Missouri 65211
}

\begin{abstract}
The effect of the worry and emotionality components of test anxiety was examined in a picture recognition task. Subjects performed orienting tasks that required a forced-choice decision concerning some physical attribute (e.g., colorfulness) or some abstract attribute (e.g., geographic locale). The abstract-feature decisions led to better recognition on an unannounced test, compared to the physical-feature task. Test anxiety had no effect on recognition performance.
\end{abstract}

The depth-of-processing model of memory assumes that processing of the semantic (deep) features of words leads to better retention than processing of purely sensory (shallow) features (Craik, 1977). This general prediction has been supported by the results of several experiments that used incidental orienting tasks to influence the nature of stimulus processing (e.g., Hyde \& Jenkins, 1973). The present research derived from some studies that indicated that arousal level may partially determine the level of processing that occurs.

Schwartz (1975) concluded that highly aroused subjects were less likely to attend to the semantic features of words than were low-arousal subjects, with more aroused subjects relatively more likely to attend to physical features. In other words, while high-arousal subjects may show a general performance deficit relative to low arousal, the deficit may be more pronounced (or present only) for semantic processing. One explanation for these results is that, in addition to generally restricting the range of cues utilized (Easterbrook, 1959), high levels of arousal and anxiety also affect the type or depth of feature utilized. While this may be more clear-cut for arousal manipulations and introversion-extraversion classifications (Eysenck, 1977), there is some supportive evidence with anxiety classifications as well (Mueller, 1979).

The present experiment extended the depth analysis of anxiety effects in memory to pictorial recognition. It has been shown that the depth-of-processing effect also occurs for facial recognition (e.g., Mueller, Bailis, \& Goldstein, in press). That is, performance on an unannounced recognition test is better when subjects had an orienting task that required processing of some

Requests for reprints should be sent to J.H. Mueller, Psychology Department, 210 McAlester Hall, University of Missouri, Columbia, Missouri 65211. This research was supported in part by funds from the Research Council of the Graduate School of the University of Missouri. The authors wish to acknowledge Gary Cooper for the provision of the slides used in this study, and Al Goldstein for providing helpful comments on an earlier draft. abstract trait (e.g., honesty) than when the task involved some physical feature (e.g., lip thickness). Furthermore, the general anxiety deficit that occurs for verbal recall also occurs for facial recognition, consistent with the assumption of reduced or differential cue utilization under high anxiety. Specifically, if high-anxiety subjects normally process shallow features, and if shallow features are less durable than deep features (Craik, 1977), then this result would be expected. However, there is no differential effect of anxiety due to type (depth) of orienting task. That is, a deep orienting task does not overcome the deficit for high-anxiety subjects.

One recent study (Miller, Mueller, Goldstein, \& Potter, 1978) examined test anxiety and depth of the study task using landscape pictures as the stimuli, to test the generality of the effect of anxiety on pictorial recognition. Deep processing was induced by asking, "Which picture was taken in the USA?" for pairs of slides. This required subjects to process abstract features rather than merely verbally labeling the picture (e.g., "waterfall"), and such judgments led to better recognition on an unannounced test than questions focusing on purely physical properties (e.g., "Which picture is more colorful?"). There were no main effects due to test anxiety level. There was an interaction of test anxiety and task level for criterion or bias (in signal detection theory terms), as high-anxiety subjects showed more cautious responding after abstract study tasks, relative to low-anxiety subjects, but vice versa after physical study tasks. It was speculated that this pattern might reflect compatibility with normal modes of processing: high-anxiety subjects "less comfortable" after deep tasks since they normally process shallowly, and low-anxiety subjects less comfortable after shallow tasks since they normally process deep features. Replication of this result would be quite interesting.

This experiment was an extension of the study by Miller et al. (1978). The procedure was very similar to the earlier experiment, but there were some differences. For one thing, Miller et al. obtained very low levels of recognition performance, so the test was 
simplified here by reducing the number of distractors. The present study also recorded the results of the subject's choices during study, so that test performance for chosen-unchosen subsets could be analyzed separately. This "congruity" factor has been implicated as an important aspect in current depth-of-processing analyses of verbal recall (Craik, 1977). It is conceivable that anxiety effects would be limited to one subset or the other, thus possibly having been attenuated in the previous study. Further, the subjects were given a second test in this experiment. After the conventional recognition test where they distinguished old scenes from new scenes, the subjects were asked for each old slide whether it was the one chosen during study. It seemed that anxiety might have an effect on memory for choice, whether or not it has an effect on recognition per se.

There was also a change in the manner of subject selection. Miller et al. (1978) used the Sarason (1972) test anxiety scale for subject selection. In general, the test anxiety construct seems quite compatible with the notion of restricted cue utilization (Mueller, 1979), since the common explanation for the harmful effects of high anxiety is that these subjects begin to attend to task-irrelevant events, such as self-monitoring of internal states and social feedback (Sarason, 1975). However, there is some evidence (cf. Spielberger, Gonzalez, Taylor, Algaze, \& Anton, 1978) to indicate that test anxiety may be composed of two components, (1) autonomic arousal ("emotionality"), which may actually help performance sometimes, and (2) "worry," or self-monitoring, which generally hinders performance. The present study thus used a scale (Spielberger et al., 1978) that allowed the separation of these components in an attempt to clarify the effects of these anxiety components in picture recognition.

\section{METHOD}

\section{Subjects and Design}

Seventy-two subjects were selected from a pool of about 500 introductory psychology students who had taken the Spielberger et al. (1978) test anxiety inventory. This inventory yields subscale scores for the worry (W) and emotionality (E) components. The subjects in the E-dominant group (Group E) had E scores of at least 18, with the E score exceeding the $\mathrm{W}$ score by at least four points (mean $\mathrm{E}=26.9$, mean $\mathrm{W}=16.6$ ). The subjects in the low-low group (Group L) had both $E$ and $W$ scores less than 12 (mean $E=10.9$, mean $W=10.0$ ). For the last group, we attempted to select a W-dominant group, but it is apparently uncommon to be highly worried without being highly emotional, yet not necessarily vice versa. (This also makes it infeasible to factorially manipulate $\mathrm{W}$ and $\mathrm{E}$ levels.) Therefore, the third group might best be labeled as "both high" (Group B), with both $E$ and $W$ scores greater than 18 (mean $E=24.3$, mean $\mathrm{W}=24.2$ ).

At the beginning and end of the experiment, subjects were administered the State Anxiety Inventory (Spielberger, Gorsuch, \& Lushene, 1970) and the Thayer (1967) Affect Checklist, counterbalanced as first and second tasks. These served as a manipulation check on the test anxiety classification. The average state anxiety scores for Groups L, E, and B were 30.9, 38.5 , and 39.9, respectively $[F(2,60)=8.09, \mathrm{MSe}=69.214$, $\mathrm{p}<.001]$. The total Thayer scores were $30.1,31.8$, and 32.4 , respectively $[\mathrm{F}(2,60)=2.73, \mathrm{MSe}=12.317, \mathrm{p}<.08]$. It thus appears that the subjects initially identified as low anxiety were in fact less anxious in the experimental setting. To assure arousal of the high-anxiety subjects, the general instructions included ego-involving statements, alleging a relationship between intelligence and the experimental task (postexperimental debriefing clarified this for the subjects).

The basic experimental design thus involved the factorial combination of three anxiety groups $(\mathrm{L}, \mathrm{E}, \mathrm{B})$ and two orienting tasks (abstract or physical feature), with six men and six women per group. Since sex entered into no significant effects, this factor will not be considered further.

\section{Materials}

The stimuli were 45 color slides of naturalistic landscapes. Fifteen pairs of study slides were chosen so that each member of a pair involved the same theme (e.g., sunsets, waterfalls, etc.). For each of these pairs, there was one distractor of the same thematic content.

\section{Procedure}

The study phase was disguised as a test of the ease of making judgments about pairs of pictures. For the abstract task, subjects decided either "Which picture was taken in the USA?" or "Which picture is more aesthetically pleasing?" For the physical-feature task, subjects decided either "Which slide is more colorful?" or "Which slide has the most sunshine?" Subjects made the same forced-choice decision for all pairs, with the alternative versions of the abstract or shallow tasks used equally often in each group. Subjects were encouraged to make their decisions as rapidly as possible and to mark them on an answer sheet at their desks. The subsequent recognition test was not mentioned.

Subjects were run in small groups. The 15 study pairs were presented at a 5 -sec rate. The test phase began about $3 \mathrm{~min}$ after the last pair of study slides. The 30 study slides were randomly mixed with 15 distractors. These were presented one at a time at a 5-sec rate, with subjects marking an answer sheet to indicate whether they thought each one had been shown before. They were not limited to only 30 "old" responses, and no mention was made of guessing (either to encourage it or imply penalties).

Finally, after a 4- to 5-min delay, the subjects were shown the 30 study slides one at a time at a 5 -sec rate, in random order. They were required to indicate for each one whether they thought it was the member of a pair they had chosen when making the orienting judgment.

\section{RESULTS}

\section{First Test}

Table 1 summarizes performance on both tests. In addition to hit rate and false alarm rate, signal detection theory measures were also computed for the first test. The analysis for the first test data involved a 2 by 3 by 2 mixed-factorial design, for task depth, anxiety group, and study response (chosen, unchosen), with the last factor within subjects.

The anxiety main effect was not significant ${ }^{1}$ for any of these measures (Fs < 1.07), nor did this factor enter into any significant interactions. Abstract decisions led to higher hit rates and $\mathrm{d}^{\prime}$ than physical-feature judgments $[\mathrm{Fs}(1,60)=11.36$ and $14.45, \mathrm{MSe}=.022$ and .890 , respectively] , and there was no task-depth effect 
Table 1

Recognition Performance on the First and Second Tests by Anxiety Group and Type of Orienting Task

\begin{tabular}{|c|c|c|c|c|c|c|}
\hline & \multicolumn{2}{|c|}{ Both High } & \multicolumn{2}{|c|}{ E Dominant } & \multicolumn{2}{|c|}{ Both Low } \\
\hline & A & $\mathbf{P}$ & A & $\mathbf{P}$ & A & $\mathbf{P}$ \\
\hline & \multicolumn{6}{|c|}{ Test 1} \\
\hline Hit Rate & .73 & .71 & .76 & .65 & .80 & .68 \\
\hline False Alarm Rate & .19 & .28 & .16 & .27 & .19 & .28 \\
\hline Old Responses & 24.67 & 25.33 & 26.33 & 24.33 & 25.50 & 23.67 \\
\hline$d^{\prime}$ & 1.54 & 1.19 & 1.72 & 1.04 & 1.99 & 1.25 \\
\hline \multirow[t]{2}{*}{$\beta$} & 1.37 & 1.08 & 2.22 & 1.25 & 1.87 & 2.51 \\
\hline & \multicolumn{6}{|c|}{ Test 2} \\
\hline Correct: Chosen & 9.83 & 10.58 & 10.58 & 10.08 & 11.08 & 11.25 \\
\hline Correct: Unchosen & 9.00 & 9.42 & 10.75 & 9.67 & 10.50 & 9.33 \\
\hline
\end{tabular}

Note $-A=$ abstract,$P=$ physical .

for $\beta(F<1)$. The depth effect was also present for false alarm rates $[F(1,60)=9.01, \mathrm{MSe}=.019]$, as abstract decisions led to fewer misidentifications. There was thus no depth effect for total "old" responses (hits plus false alarms; $F<1.54$ ). Although there were no significant interactions, individual comparisons revealed that the task-depth effect for hit rate and $\mathrm{d}^{\prime}$ was significant only for Group L. The Task Depth by Anxiety interaction observed by Miller et al. (1978) for $\beta$ was not apparent here.

Slides chosen during study were better recognized than unchosen slides in terms of hit rate and $\mathrm{d}^{\prime}$ $[\mathrm{Fs}(1,60)=6.78$ and 7.38 , MSe $=.010$ and .150 , respectively]. Chosen slides also had a lower criterion than unchosen slides (means $=1.62$ and 1.75 , respectively) $[\mathrm{F}(1,60)=4.98, \mathrm{MSe}=.109]$. Choice did not enter into any significant interactions.

The nonparametric signal detection analysis (Grier, 1971) revealed statistical conclusions identical to $\mathrm{d}^{\prime}$ and $\beta$.

\section{Second Test}

Only one effect was significant on. Test 2: Slides chosen as meeting the criterion in study were more often identified as such than unchosen slides (means $=10.40$ and 9.78 correct, respectively, with a maximum of 15) $[F(1,60)=4.48$, MSe $=3.14]$. The task-depth effect was not significant $(F<1.36)$, nor was the anxiety group effect $(F<1.26)$.

\section{Additional Analyses}

As further tests for anxiety effects, the subjects were divided at the median first on their state anxiety scores, and second on their Thayer scores, and tests were then run in each case. The purpose of such subject reclassification is that test anxiety selects subjects with predispositions for anxiety, and although the test anxiety subgroups differed as groups on state anxiety, it is still possible that some subjects identified as anxious were not in fact aroused by the experiment, and vice versa for low-anxiety subjects. However, these analyses failed to reveal significant anxiety differences for any of the measures discussed above. Table 2 presents the results of correlations computed between the various anxiety measures and the recognition measures. Virtually none reached acceptable levels of significance.

\section{DISCUSSION}

The present results replicate two essential features of the previous study by Miller et al. (1978). First, the task-depth factor produced the expected result, although it was most apparent for low-anxiety subjects. Second, anxiety produced no main effect or interactions. It is apparent in Table 1 that Group B did generally perform a little worse than Group L, the comparison most like the conditions of the previous study, but the differences were not significant. Thus, this result seems to have some generality in view of the differences between the present study and the previous one (e.g., task difficulty and the anxiety scale used for selection of subjects). Whatever the reason, it appears that anxiety may not generally interfere with pictorial recognition, although it may hinder facial recognition (Mueller et al., in press) and regularly hinders verbal recall (Mueller, 1979).

The use of W and E subscales was not particularly helpful here, in spite of the fact that the size of the original pool of subjects used for selection should have provided an ample range of scores. This may be due to the apparent empirical interdependence of these components. In other words, while it may be conceptually possible to distinguish $\mathrm{W}$ and $\mathrm{E}$ components, in practical terms these covary. In terms of relative performance here, Group E generally fell in between Groups B and $L$, and there was no evidence of facilitation by high levels of E. Admittedly, Group E subjects also had fairly high W scores too, due to the interdependence. To pursue this further, the raw $\mathrm{W}$ and raw $\mathrm{E}$ scores were correlated with the recognition measures. As Table 2 shows, the correlations were quite low, but most important, the correlations were virtually all negative for both $\mathrm{E}$ and $\mathrm{W}$. That is, there was no evidence of a pattern of positive (or nonsignificant) correlations between E scores and the performance measures accompanied by negative correlations for W scores. If anything, higher levels of both seemed to be associated with worse recognition performance, although nonsignificantly so.

It is possible that this is a problem limited to the present procedures and materials. Specifically, it appears that pictures of landscapes may not be very sensitive to anxiety differences in general. If this is true, then these experiments may not offer a fair test of the differential properties of the $\mathrm{W}$ and $\mathrm{E}$ anxiety

Table 2

Correlations Between Test Anxiety Scores, State Anxiety, Thayer General Activation Scores, and Recognition Performance

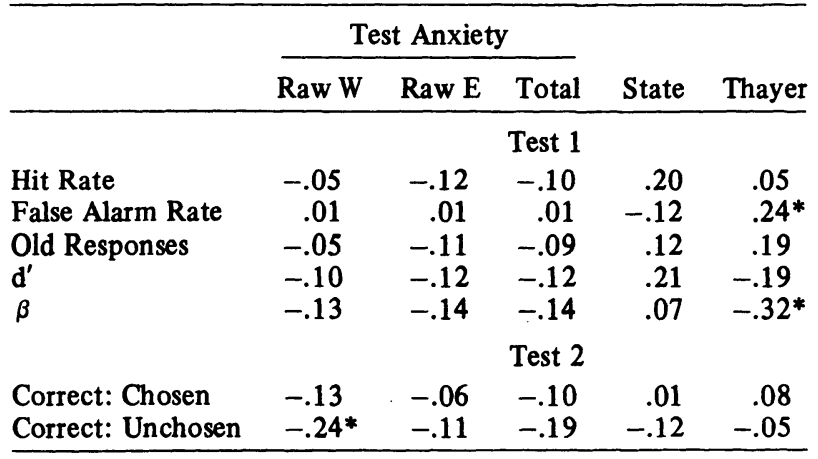

${ }^{*} p<.05$. 
components. Therefore, it may be premature to abandon an appealing conceptual distinction at this point. However, it does seem established that degradation of performance by high anxiety does not occur with such pictorial materials, and the distinction between verbal and pictorial materials may be useful in terms of further research on the effects of anxiety on memory.

\section{REFERENCES}

CRAIK, F. I. M. Depth of processing in recall and recognition. In S. Dornic (Ed.), Attention and performance (Vol. 6). Hillsdale, N.J: Erlbaum, 1977.

EASTERBROOK, J. A. The effect of emotion on cue utilization and the organization of behavior. Psychological Review, 1959, 66, 183-201.

EYSENCK, M. W. Human memory: Theory, research and individual differences. London: Pergamon Press, 1977.

GRIER, J. B. Nonparametric indexes for sensitivity and bias: Computing formulas. Psychological Bulletin, 1971, 75, 424-429.

HYDE, T. S., \& JENKINS, J. J. Recall for words as a function of semantic, graphic, and syntactic orienting tasks. Journal of Verbal Learning and Verbal Behavior, 1973, 12, 471-480.

Miller, D. J., Mueller, J. H., Goldstein, A. G., \& Potter, T. L. Depth of processing and test anxiety in landscape recognition. Bulletin of the Psychonomic Society, 1978, 11, 341-343.
Mueller, J. H. Test anxiety and the encoding and retrieval of information. In I. G. Sarason (Ed.), Test anxiety: Theory, research, and applications. Hillsdale, N.J: Erlbaum, 1979.

MuelleR, J. H., Bailis, K. L., \& Goldstein, A. G. Depth of processing and anxiety in facial recognition. British Journal of Psychology, in press.

SARAson, I. G. Experimental approaches to test anxiety: Attention and the uses of information. In C. D. Spielberger (Ed.), Anxiety: Current trends in theory and research (Vol. 2). New York: Academic Press, 1972.

SARASON, I. G. Anxiety and self-preoccupation. In I. G. Sarason \& C. D. Spielberger (Eds.), Anxiety and stress (Vol. 2). Washington, D.C: Hemisphere/Wiley, 1975.

SchwARTz, S. Individual differences in cognition: Some relationships between personality and memory. Journal of Research in Personality, 1975, 9, 217-225.

Spielberger, C. D., Gonzalez, H. P., Taylor, C. J., Algaze, B., \& Anton, W. D. Examination stress and test anxiety. In C. D. Spielberger \& I. G. Sarason (Eds.), Anxiety and stress (Vol. 5). Washington, D.C: Hemisphere/Wiley, 1978.

SPIElberger, C. D., Gorsuch, R. L., \& LuShene, R. E. Manual for the State-Trait Inventory. Palo Alto, Calif: Consulting Psychologists Press, 1970.

THAYER, R. E. Measurement of activation through self-report. Psychological Reports, 1967, 20, 663-678.

(Received for publication January 16, 1979.) 